\title{
The Computer Code for Calculations of the Positron Distribution in a Layered Stack Systems
}

\author{
K. SIEMEK ${ }^{a, *}$ AND J. DRYZEK ${ }^{a, b}$ \\ ${ }^{a}$ Institute of Nuclear Physics PAS, E. Radzikowskiego 152, 31-342 Kraków, Poland \\ ${ }^{b}$ Institute of Physics, Opole University, Oleska 48, 45-052 Opole, Poland
}

\begin{abstract}
In this paper we present experimental and theoretical studies of the implantation profile of positrons emitted from $^{22} \mathrm{Na}$ radionuclide into layered sample. The measured profile for the nonsymmetrical, around the positron emitter, stack of aluminium and silver foils was measured using the depth scanning of implantation profile technique. For the description of the obtained results two theoretical approaches were applied. The first one was the Monte Carlo simulation using the GEANT4 tool kit. The generated profile does not reproduce accurately the experimental profile. We proposed the multiple scattering model based on the fact that a positron due to backscattering can travel as an energetic particle several times through the whole sample before it annihilates. In this model absorption is also taken into account. The multiple scattering model algorithm was implemented in the LYS-1 program. The calculated profile using the LYS-1 program reproduces the experimental profile with much better accuracy.
\end{abstract}

DOI: 10.12693/APhysPolA.125.833

PACS: 78.70.Bj, 52.25.Tx

\section{Introduction}

A positron injected to matter loses its kinetic energy by collisions with nuclei, electrons and phonons or by emission of bremsstrahlung photons, until finally reaching thermal energy. The distance it travels is mainly defined by its initial energy and properties of implanted material. After thermalization a positron randomly walks and penetrates a volume occupied by around $10^{9}$ atoms in a sample, finally it annihilates with an electron. Commonly, positrons are implanted from radionuclides into the solid with high energies and finally a certain depth distribution, called the implantation profile, is constituted. The positron implantation profile affects the measured annihilation characteristics like a positron lifetime spectrum, or mean positron lifetime. This is valid for slow positron beam experiments, but also for conventional positron spectroscopy, for instance the so-called source contribution is defined by this profile.

However, one can consider a sample in the form of stack which consists of layers or foils illuminated by positrons. The distribution of positrons in such a sample can be deduced from positron lifetime measurements. Theoretical calculations based on Monte Carlo (MC) simulations using the GEANT4 code allow us to obtain the intesity of lifetime components in the positron lifetime spectrum as well. However, they have shown significant differences with experimental data, for the case when foils are distributed nonsymmetrically around the positron source [1]. Also, direct measurements of the positron implantation profile using the depth scanning of positron implantation profile (DSIP) technique pointed out that

*corresponding author; e-mail: krzysztof.siemek@ifj.edu.pl the MC simulation are not able to describe the experimental results accurately. In this work we present new theoretical model, called the multiple scattering model (MSM) [2], which can be exploited in layered samples to enable fast calculation of implantation profiles and lifetime component intensities. Flexibility of the model allows accurate reproduction of the measured data even for the nonsymmetrical stack of foils distributed around the positron source. We report the comparison of the positron implantation profile calculated using the MSM, the simulated profile using the GEANT4 code and the experimental profile measured using the DSIP technique for the stack which consisted of $\mathrm{Al}$ and $\mathrm{Ag}$ foils.

\section{Multiple scattering model}

The MSM considers two phenomena: positron absorption in layers and backscattering at the interfaces between them. It assumes that due to backscattering a positron can travel as an energetic particle through the whole sample several times before it thermalizes and annihilates, see Fig. 1. For simplification trajectories in the layers are considered as straight lines. Positron diffusion is neglected in the consideration because the thickness of the layers is several $\mu \mathrm{m}$. The absorption process is described by the transmittance function $I\left(x, \alpha^{+}\right)$, which defines the probability that a positron passes a distance $x$ away from the source. The MC simulations indicated that this function can be described by two exponential functions as follows:

$$
\begin{aligned}
& I\left(x, \alpha^{+}\right)= \\
& \left\{\begin{array}{c}
\frac{N}{2}\left[\exp \left(-2 \alpha^{+} x\right)+\exp \left(-2 \alpha^{+} d_{0}\right)\right], \quad x \leq d_{0}, \\
N \exp \left(-\alpha^{+}\left(x+d_{0}\right)\right), \quad x>d_{0},
\end{array}\right.
\end{aligned}
$$

where $N=2 /\left[1+\exp \left(-2 \alpha^{+} d_{0}\right)\right]$ is the normalized constant, $\alpha^{+}$is the coefficient of absorption and $d_{0}$ is the 
function parameter. From the MC simulations it was established that $d_{0} \simeq 0.5 / \alpha^{+}$. This function describes the profile in case of a semi-infinite sample; however, it does not include backscattering of positrons at interfaces which occurs in layered samples.

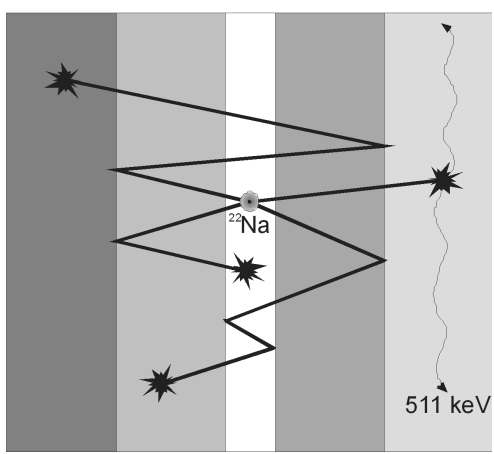

Fig. 1. Demonstration of four positron tracks in layered samples considered in the MSM.

Due to backscattering at the interfaces the motion of positrons in the stack of foils or layers is complicated. For finding their distribution an iterative procedure must be applied. In the first step only absorption of positrons moving towards the layer is accounted for. However, when backscattering occurs positrons can be absorbed also travelling back to the source or reach the support source layer again which gives the next iteration step. When positrons travel back to the source layer, they are treated as new positrons source, localized at interfaces between sample and the source support foil. The procedure is repeated and includes fractions of positrons backscattered until the desired accuracy of the calculations is achieved, for which the number of positrons does not exceed $10^{-7}$ of the number of initial positrons. Then the total transmittance function is the sum of the first and next iteration steps. Calculations of implantation profile $P(x)$ is done using the derivative of the total number of positrons reaching the distance $x$ from the source. More details about the MSM can be found in Ref. [2]. The algorithm of the MSM was implemented in the program LYS-1 (LaYerS profile analysis) and is easy available online [3]. In this model, the only input parameters used to determine the positron distribution and the profile are the backscatter and absorption coefficients of particular layers and the energy end-point of the Fermi distribution of $\beta^{+}$decay $E_{\max }$, which is well known for each $\beta^{+}$radioisotope. A coefficient of absorption and backscattering can be calculated based on several empirical formulae [4-9]. For example, in order to determine the backscatter coefficient a formula developed by MacKenzie et al. [4] can be used

$$
R=0.342 \log _{10} Z-0.146,
$$

where $Z$ - average atomic number $Z$ of material.

On the other hand, a good formula to determine the absorption coefficient of positrons was proposed by
Dryzek [8]:

$$
\alpha^{+}=12.6 \frac{Z^{0.17}}{E_{\max }^{1.28}} \rho\left[\mathrm{cm}^{-1}\right],
$$

where $\rho$ is the density of the material of a layer expressed in $\mathrm{g} / \mathrm{cm}^{3}$, and $E_{\max }$ in $\mathrm{MeV}$. With knowledge of these parameters calculations of implantation profile can be performed.

One should emphasize that the support foils of the source are treated as a layer in the stack, then the LYS-1 program calculates the fraction of positrons annihilated in the source. Then it is useful for the calculation of the source correction necessary for the positron lifetime deconvolution procedure. The friendly interface and the clear graphic presentation of results in the LYS-1 program allow a user to determine the positron distribution in a complex stack which consists of nine layers. In the code a fast algorithm was applied and the results are available in seconds.

\section{Experimental}

For measurement of the positron implantation profile the DISP is a suitable technique $[10,11]$. In this technique a sample is placed in front of the slit between two thick lead blocks, see Fig. 2. The slit is $60 \mu \mathrm{m}$ thick. A coaxial $\mathrm{HpGe}$ detector is placed behind the slit. A micrometer screw allows shifting the sample in the direction perpendicular to the slit. An HpGe detector measures the energetic spectrum of the annihilation line at $511 \mathrm{keV}$. The number of counts under the annihilation line is proportional to the number of positrons annihilating at the selected depth from the positron source. The

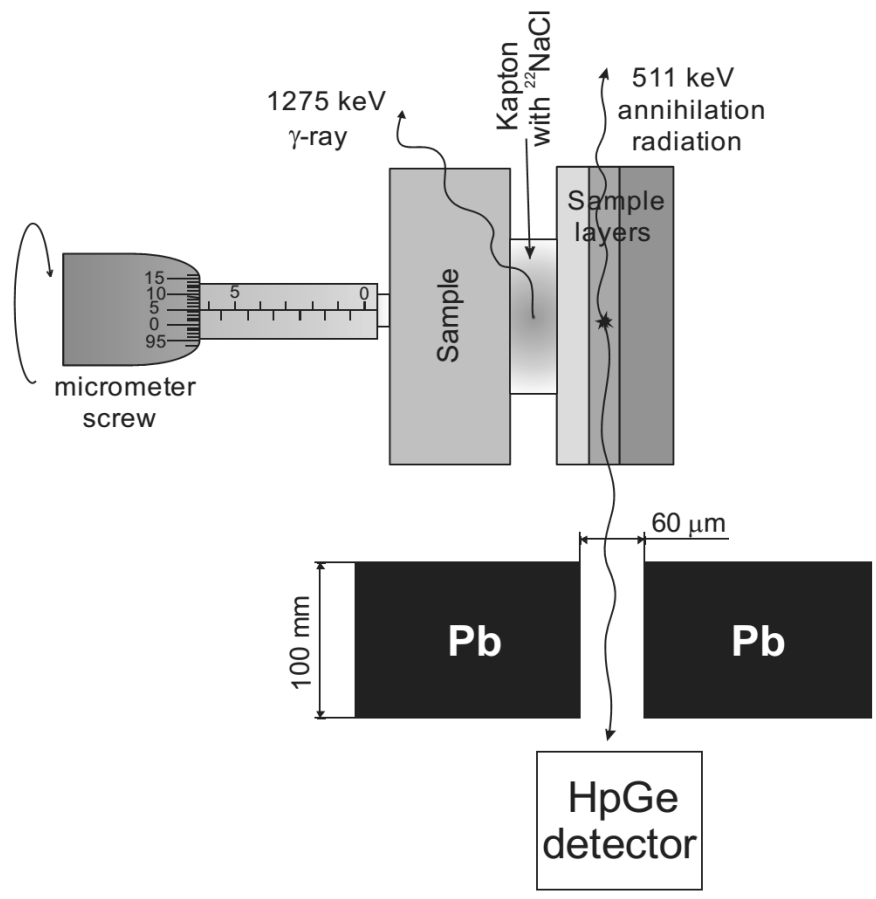

Fig. 2. The scheme of the DSIP technique for measurement of the positron implantation profile. 
measurement accuracy depends on the width of the slit through which the annihilation photons reach the HpGe detector. This method allows to measure positron implantation profile also for layered samples.

Our studies were carried out with the stack of plates and foils located non-symmetrically around of the positron emitter. The ${ }^{22} \mathrm{Na}$ isotope enveloped between two $7 \mu \mathrm{m}$ kapton foils is the emitter. On its left side the $2 \mathrm{~mm}$ thick aluminium plate was placed. On the other side, there were the aluminum foil $100 \mu \mathrm{m}$ thick, then the $40 \mu \mathrm{m}$ thick silver foil and the $2 \mathrm{~mm}$ thick aluminum plate, i.e., $\mathrm{Al}(2 \mathrm{~mm})-{ }^{22} \mathrm{Na}-\mathrm{Al}(100 \mu \mathrm{m})-$ $\mathrm{Ag}(40 \mu \mathrm{m})-\mathrm{Al}(2 \mathrm{~mm})$, see inset in Fig. 2. The two aluminum plates on the edges of the stack served role of a target and also to compress the thin foils. Before the experiment the foils were annealed to avoid localization of positrons in defects present in the samples.

\section{GEANT4 simulations}

In the simulations we created the geometrical stack which was a reproduction of the experimental configuration. Only the thickness of the positron source was changed to $50 \mu \mathrm{m}$ in comparison with the thickness of kapton foil, because presence of glue and radionuclide compound makes source much thicker. The value $50 \mu \mathrm{m}$ corresponds to the real dimension of the source. The source layer in the simulations was treated as kapton foil with a point positron source ${ }^{22} \mathrm{Na}$ in the middle of this foil. First we make simulations using GEANT4 code [12]. GEANT4 realizes the Monte Carlo method, which allows tracking and scattering processes in wide energy range. It includes physical phenomena such as the photoelectric effect, the Compton and Rayleigh scattering, bremsstrahlung, ionization, secondary electron emission, the Auger effect and others. The simulation tracked about one million positrons to get results suitable for further analysis. The simulation accuracy was $10^{-6}$. Results of simulations were normalized to the maximum of the implantation profile and presented as a gray line in Fig. 3.

In the left part of the stack, where the aluminum plate is located, a smooth, almost exponential decay is obtained, Fig. 3. However in the right part, the discontinuities at the interfaces between the foils are observed - mainly, the sharp increase at the border between the aluminum and silver foil. Also, the interface between the source and the aluminum plate and foils is sharply visible. This is an effect of backscattering of positrons at the interfaces between media of different physical properties.

\section{The MSM calculations}

The second approach was to use the LYS-1 program where the MSM were implemented. At first, as an input we applied the backscattering and absorption coefficients calculated from Eqs. (2) and (3). The profile obtained in this way is presented in Fig. 3 as a black dashed

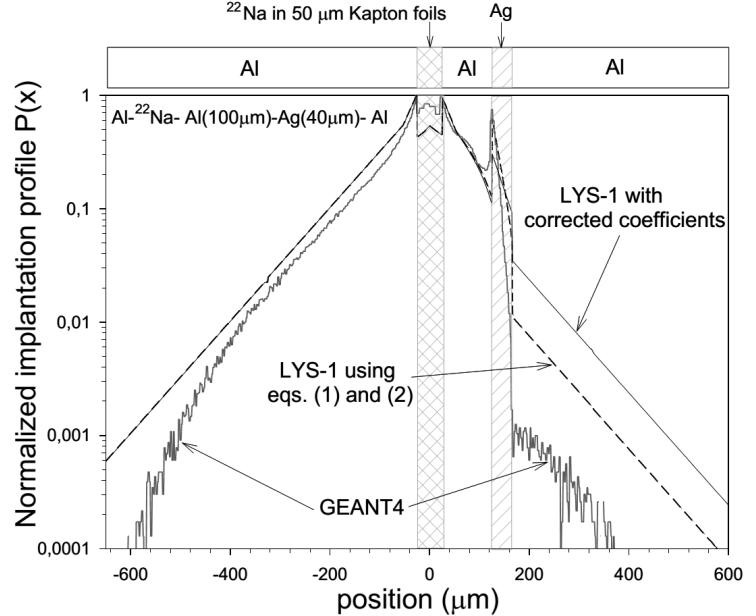

Fig. 3. The net implantation profiles for ${ }^{22} \mathrm{Na}$ positrons emitted into the nonsymmetrical stack of foils around the source. The stack composition is shown in the top. The dark gray line shows the positron implantation profile obtained from MC simulations using GEANT4 code. The dashed black line presents the implantation profile obtained using the MSM and Eqs. (2) and (3) implemented in the LYS-1 program. The black line shows the corrected MSM profile with adjusted parameters.

line. Here, steps at the interfaces between foils are also clearly visible; however, one can see differences between the LYS-1 results and GEANT4 profile. The main dissimilarity is in the source and silver foils areas. GEANT4 predicts more positron absorption in the silver foil and less in the source than the LYS-1 program. Nevertheless, the results obtained by LYS-1 program follow the form of those from GEANT4.

\section{Comparison with experiment}

The results of measurements, showing the implantation profile as a function of distance from the radionuclide, are presented on a logarithmic scale in Fig. 4 as open circles. The maximum of the profile is observed at the position where positron emitter is placed, then number of counts decreases with increasing distance from source. We can recognize the exponential decay in the left part of the stack where only aluminum plate is located. In the right part, where the foils are present, the profile decays but the dependence exhibits a much more complex shape. In the next step we try to describe the obtained profile using two methods, the MC simulations using GEANT4 and the MSM calculations.

In order to compare directly the obtained profiles with the experimental one we should convolute them with the spatial resolution function. We chose the Gaussian function with half-width corresponding to the width of the slit, and a background was added to the theoretical profile using the signal-to-noise ratio of 400:1. The solid lines in Fig. 4 present the results of the convolution procedure 


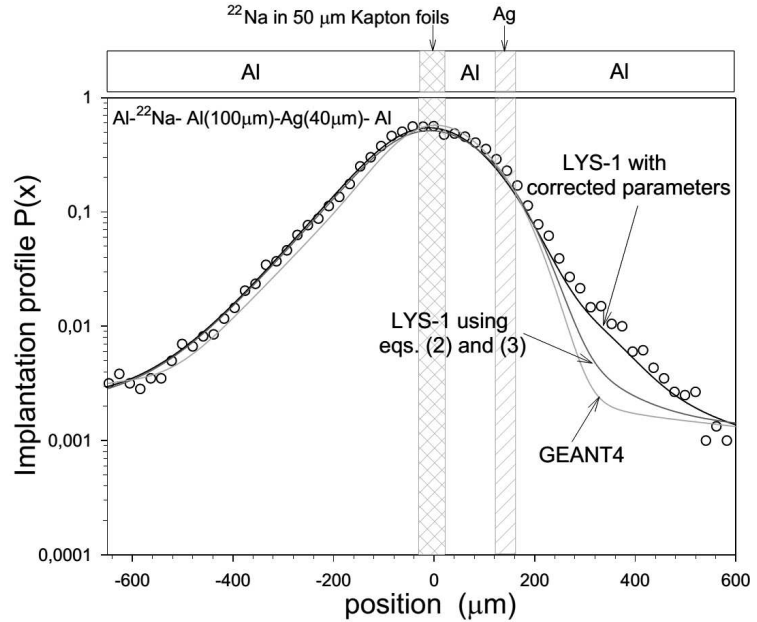

Fig. 4. The positron implantation profile (open circles) measured using the DSIP technique for the nonsymmetrical stack of foils around the source. The stack composition is shown in the top. The light gray line presents the positron implantation profile obtained from MC simulations using GEANT4 code. The dark gray line is the implantation profile obtained using the MSM and Eqs. (2) and (3) implemented in the LYS-1 program. The black line shows the corrected MSM profile with adjusted parameters. All three profiles were taken from Fig. 3 and the convolutions were performed with the Gaussian function, see the text.

for the theoretical profiles taken from Fig. 3. The light gray line corresponds to the profile obtained using the GEANT4 simulations and the gray line - to the profile obtained using the LYS-1 program. One can see the good agreement between the simulated and calculated profiles with the experimental one, but only in the left part. However, in the right part a discrepancy is noticed. In order to find the better agreement we generated the profile using the LYS-1 program and we corrected the absorption coefficient of silver foil from 553 to $300 \mathrm{~cm}^{-1}$ and increased the backscatter coefficient from 0.43 to 0.5 . The obtained profile is presented in Fig. 3 as a solid black line, and after convolution with the Gaussian function also as a solid line in Fig. 4. Now good agreement with the experimental points across the whole stack is achieved.

We should remark that the absorption coefficient given in Eq. (2) was determined in the case when the positrons were implanted into a semi-infinite solid sample. One can suppose that for thin foils, due to the backscattering effect, this coefficient can be modified. We argue that the LYS-1 program can help to investigate this effect.

\section{Conclusion}

The developed LYS-1 program with implemented MSM turned out to be a good tool kit for the calculations of the implantation profile of energetic positrons in layered samples. We found good agreement between the calculated profile and the experimental implantation profile measured using the DSIP technique for the stack of plates and foils of aluminium and silver positioned non-symmetrically around the positron emitter. However, the input coefficients for the LYS-1 program, i.e., the linear absorption and backscattering coefficients for the silver foil, were changed from their bulk values. The absorption coefficient of silver foil was equal to $300 \mathrm{~cm}^{-1}$ and the backscatter coefficient 0.5 ; the bulk values are equal to $553 \mathrm{~cm}^{-1}$ and 0.43 , respectively. We found also that the MC simulations using GEANT4 cannot reproduce the experimental implantation profile in the stack with an accuracy as good as that calculated by the LYS-1 program.

\section{References}

[1] J. Dryzek, P. Horodek, Nucl. Instrum. Methods Phys. Res. B 269, 1411 (2011).

[2] J. Dryzek, K. Siemek, J. Appl. Phys. 114, 074904 (2013).

[3] K. Siemek, www.ifj.edu.pl/ mdryzek/page_r22.html .

[4] I.K. MacKenzie, C.W. Schutle, T. Jackmann, J.L. Campbell, Phys. Rev. A 7, 135 (1973).

[5] J. Dryzek, E. Dryzek, Phys. Lett. A 320, 238 (2003).

[6] P.U. Arifov, A.R. Grupper, H. Alimkulov, Positron Annihilation, Ed. P.G. Coleman, North-Holland, Amsterdam 1982, p. 699.

[7] Nathu Ram, I.S. Sundara Rao, M.K. Mehta, Pramana 18, 121 (1981).

[8] J. Dryzek, D. Singleton, Nucl. Instrum. Methods Phys. Res. B 252, 197 (2006).

[9] G.I. Gleason, I.D. Taylor, D.L. Tabern, Nucleonics 8, 12 (1951).

[10] J. Dryzek, P. Horodek, Nucl. Instrum. Methods Phys. Res. B 267, 3580 (2009).

[11] J. Dryzek, Appl. Phys. A 81, 1099 (2005).

[12] GEANT4 web page: cern.ch/geant4 . 